\title{
e-Migrinter
}

$8 \mid 2012$

Regards sur les migrations sud-asiatiques

\section{Transformations urbaines et Migrations dans les villes espagnoles}

Compte rendu des journées d'études organisées à Poitiers les 8 \& 9

décembre 2011

Marine Bertrand

\section{(2) OpenEdition}

Journals

Édition électronique

URL : https://journals.openedition.org/e-migrinter/654

DOI : 10.4000/e-migrinter.654

ISSN : 1961-9685

Éditeur

UMR 7301 - Migrinter

Édition imprimée

Date de publication : 4 avril 2012

Pagination : 124-127

ISSN : 1961-9685

Référence électronique

Marine Bertrand, "Transformations urbaines et Migrations dans les villes espagnoles », e-Migrinter [En ligne], 8 | 2012, mis en ligne le, consulté le 20 mai 2021. URL : http://journals.openedition.org/emigrinter/654 ; DOI : https://doi.org/10.4000/e-migrinter.654 


\section{«Transformations urbaines et Migrations dans les villes espagnoles ». Journées d'études organisées à Poitiers les $8 \& 9$ décembre 2011}

$\mathbf{L}$ es 8 et 9 décembre 2011, deux journées d'étude portant sur le thème des « transformations urbaines et migrations dans les villes espagnoles " se sont déroulées dans les locaux de la MSHS de l'Université de Poitiers. Organisée par Naïk Miret (Migrinter), cette rencontre a rassemblé pour la quatrième fois consécutive des chercheurs de plusieurs disciplines.

\section{Compte rendu par Marine Bertrand}

Au travers de cas de villes espagnoles marquées par l'arrivée massive de migrants internationaux depuis la décennie 1990, les intervenants ont interrogé concepts, catégories et outils permettant de penser les relations entre les pratiques spatiales des migrants et la production et la transformation des villes. Comment observer l'insertion de ces «nouveaux voisins » dans l'espace urbain ? Comment identifier les permanences et les ruptures dans l'inscription territoriale des migrants dans les villes? Comment, par leurs mobilités, leurs pratiques spatiales et leurs relations avec les «autochtones», participent-ils à fabriquer la ville ? Comment l'étude de ces dynamiques migratoires et urbaines permet-elle d'éclairer d'autres processus et mécanismes sociaux?

Trois intervenantes se sont penchées sur les processus d'inscription territoriale d'un groupe de migrants particulier à l'échelle d'une ville. Lucile Medina, géographe à l'Université de Montpellier, a porté son regard sur les logiques résidentielles de migrants boliviens à Madrid et, plus amplement, sur les processus de construction de lieux et de territoires migrants à travers l'utilisation et l'appropriation des espaces ainsi que par les «régimes de visibilité » de ce groupe. Trois critères, à savoir les usages sociaux et culturels des lieux, leurs poids en termes d'identification du groupe et leur rôle de centralité ethnique ou multiculturelle, lui ont permis de construire une typologie des territoires boliviens dans Madrid. Elle propose la distinction de trois types de territoires: les quartiers d'invisibilité ethnique, les quartiers multiethniques et les centralités boliviennes. Une démarche de 
micro-géographie se révèle ainsi indispensable pour étudier la visibilité du groupe migrant à partir des processus de construction de lieux.

Les migrants boliviens font également objet de l'attention de Susana Sassones, géographe à l'Instituto Multidisciplinar de Historia y Ciencias Humanas (CONICETIMHICIHU) de Buenos Aires, qui interroge ensemble les logiques de circulation et de migration de cette population (dans leurs dimensions historique et sociodémographique) et leurs logiques d'inscription spatiale à Buenos Aires. Son approche, qui prend en considération les trajectoires migratoires des individus, lui permet d'observer que les mobilités résidentielles de ce groupe de migrants révèlent une double logique spatiale de concentration et de dispersion dans la ville. Alors que certains quartiers peuvent être qualifiés d' "enclaves migratoires », il est aussi possible d'observer un scénario de multi-localisation avec une tendance à des regroupements en micro-zones s'organisant en fonction du lieu d'origine des personnes.

Enfin, Marème Niang, doctorante en géographie au laboratoire ART-Dev de l'Université de Montpellier, a porté son attention sur les logiques socio-spatiales de Gambiens et de Sénégalais à Barcelone. Elle nous a livré les premiers résultats de sa recherche doctorale concernant les stratégies résidentielles de ces groupes. Le croisement des données statistiques du padrón municipal avec les observations produites dans le cadre d'une enquête qualitative, lui permettent de questionner le recours aux informations statistiques sur la résidence des personnes par nationalité, quand les pratiques spatiales semblent se différencier selon les groupes ethniques d'appartenance. L'intervenante dresse une typologie très diversifiée des pratiques résidentielles de ces migrants en conjuguant trois facteurs: les réseaux sociaux ethniques ou communautaires, le contexte spatial (à savoir, l'Aire Métropolitaine de
Barcelone) et l'origine urbaine ou rurale des personnes.

Prolongeant cette démarche d'analyse à l'échelle d'une métropole, Pau Serra del Pozo, géographe à l'Université Internationale de Catalogne, a centré son attention sur les entrepreneurs ethniques. Par un travail critique sur les concepts mobilisés pour décrire la répartition spatiale des commerces ethniques, il rejette celui d' " enclave ethnique », inapproprié selon lui aux dynamiques des villes européennes, pour lui préférer la notion de «centralité ethnique ». Il propose autour de ce concept une typologie des paysages ethniques des villes. Basée sur une mesure quantitative, cette typologie se veut un outil de comparaison possible entre différentes villes et au cours du temps. Cinq types de centralités apparaissent : a) les centralités coethniques spécialisées (un seul type de commerce et une seule nationalité d'origine des commerçants), b) les centralités coethniques diverses (différents types de commerces et une seule nationalité d'origine des commerçants), c) les centralités multiethniques (différents types de commerces et différentes nationalités d'origines des commerçants), d) dispersion métropolitaine (concentration à très petite échelle dispersée au sein de la métropole), e) enclave ethnique (bien qu'elle n'apparaisse pas à Barcelone).

Par le biais d'une observation multisituée, Tanja Bastia, chercheuse en développement urbain à l'Université de Manchester, propose d'interroger les migrations internationales comme un espace de reconfiguration des relations de genre. Pour cela, elle suit une population originaire d'une région minière de Bolivie dans différents lieux de sa migration, à Cochabamba, à Buenos Aires et en Espagne. Elle peut ainsi prendre en considération le contexte d'origine des populations, les circulations et les pratiques transnationales des personnes. Elle développe alors une approche intersectionnelle qui lui permet de se demander comment les relations de genre, 
de classe et d'ethnicité sont renégociées au travers de migrations internes et internationales. Les résultats présentés montrent une réorganisation de la division sexuelle des tâches au sein des ménages provoquée par une migration, mais une réorganisation pensée comme temporaire par les acteurs. De la même manière, s'il est possible d'observer à un niveau individuel une plus grande indépendance des femmes au travers de la migration, une analyse interscalaire et intersectionnelle suggère que les femmes échangent leurs gains dans les rapports de genre contre une mobilité sociale ascendante dans les rapports hiérarchiques de classes, contribuant ainsi, non pas à une 'libération' des femmes, mais au renforcement des relations de genre patriarcales.

\section{Le sociologue Francisco Torres} (Université de Valencia) se démarque par une approche qui ne part plus d'un groupe de migrants mais de l'espace, et plus précisément des espaces publics. Ces derniers se révèlent être, selon lui, une scène privilégiée pour l'observation des dynamiques générées par les processus d'insertion des migrants dans les villes. La présence de ces «nouveaux voisins » implique des changements et des resignifications des espaces de la ville, tant pour les migrants que pour les autochtones. Le sociologue s'interroge donc sur les usages et l'appropriation des espaces publics par ces nouveaux habitants, qui les utilisent comme des ressources de sociabilité. Cet usage des espaces publics, lieux « ouverts à tous", peut cependant créer des tensions lorsqu'il se distingue trop du modèle d'urbanité hégémonique. C'est le cas par exemple des espaces ethnicisés: appropriés par un groupe ethnique particulier, l'espace se transforme pour devenir un espace ressource de sociabilité pour ce collectif. L'image qu'en ont les autres habitants s'en trouve alors modifiée et souvent, comme le fait remarquer l'intervenant, ces lieux sont pensés comme des espaces dégradés ou de tensions. Il est nécessaire de questionner cette appréciation négative de ces lieux. Quel rôle joue l'appropriation des espaces dans l'insertion des migrants à la société d'accueil? De plus, les espaces publics «partagés » et les espaces publics ethnicisés ne sont-ils pas compatibles dans une ville? Cette question peut se poser à l'échelle d'un lieu: selon les temporalités, un espace peut être utilisé différemment. Mais également à l'échelle d'une ville : ne peut-il pas y avoir au sein d'une ville une diversité d'espaces publics? Ces questions doivent être pensées à la lumière d'une autre interrogation soulevée par l'intervenant. Les deux droits que sont le droit à l'anonymat, à l'indifférence et le droit de (re)-créer son propre espace sont-ils compatibles ? Et pour quelles personnes?

Enfin, les interventions de Naik Miret, géographe à Migrinter, et de Fatiha Belmessous, historienne au laboratoire ENTPE-RIVES de Lyon, permettent une réflexion transversale aux communications précédentes. Dans une approche rétrospective sur ses propres travaux de recherche, la première offre un regard critique sur différentes manières d'aborder les liens entre dynamiques de la ville et migrations internationales, que se soit au travers d'une entrée par le système d'immigration, par un groupe de migrants ou par l'espace. Elle remarque que le croisement de ces approches permet d'éclairer les processus de concentration et de dispersion des populations dans les villes par des processus de recomposition urbaine dans le temps long et de banaliser les expériences de substitution de peuplement dans les quartiers populaires périphériques. En écho aux présentations sur les espaces et centralités ethniques, elle invite à redécouvrir la notion de «sas » développée par l'Ecole de Chicago et à découvrir celle d' «urbanisme transnational» développée par Peter Smith. Cette notion permet de penser comment un groupe transporte dans sa migration un modèle de représentations et de pratiques de l'espace et le reproduit dans les villes où il s'installe. Fatiha Belmessous 
étudie les effets des politiques publiques sur les logiques sociales des territoires urbains qu'elle met en lumière en adoptant une perspective diachronique. Elle montre ainsi comment les politiques de logement et d'aménagement des quartiers dits "sensibles» ont contribué au changement social et à la construction de ces quartiers comme objet de recherche. En introduisant et concluant ce séminaire, elle nous a amenés à repenser l'historicité des catégories mobilisées dans l'étude des migrations internationales. Classifications administratives ou politiques ne doivent pas être confondues avec les catégorisations mises en place par les chercheurs. De même, celles-ci doivent être utilisées en prenant en compte leur processus de construction et ce, notamment si les chercheurs souhaitent s'entendre autour d'un même objet d'étude. Aussi, plusieurs concepts et catégories apparus au long de ces journées furent objet de l'exercice lors des débats.

Marine Bertrand Doctorante en Géographie Migrinter - UMR 7301 CNRS / Université de Poitiers marine.bertrand@univ-poitiers.fr

\section{Repères bibliographiques}

Baby Collin, Virginie; Medina, Lucile ; Miret, Naik ; Sassone, Susana (sous-presse) Territorios bolivianos en las metropolis españolas: Madrid y Barcelona, in Sole Puig, Carlota (coord..) Migración boliviana y prácticas transnacionales, Barcelona, Edition Antropos.

Bastia, Tanja (2011) Migration as protest? Negotiating gender, class and ethnicity in urban Bolivia, Environment and Planning, vol. 43, pp. 1514-1529.

Torres Perez, Francisco (2008) Los nuevos vecinos de la plaza. Inmigrantes, espacios y sociabilidad pública, AIBR. Revista de antropología Iberoamericana, vol. 3, $\mathrm{n}^{\circ} 3$, pp. 366-397.

Sassone, Susana ; Cortès, Geneviève (souspresse) Inmigración boliviana en la Argentina: Lógicas geográficas de difusión territorial y metropolización" in Sole Puig, Carlota (coord..) Migración boliviana y prácticas transnacionales, Barcelona, Edition Antropos.

Serra Del Pozo, Pau (2011) Dispersión y suburbanización. Nuevos paisajes étnicos, Revista Internacional de Organizaciones, $\mathrm{n}^{\circ} 6$. pp. 65-79. 